\title{
New oscillation criteria for second-order neutral differential equations with distributed deviating arguments
}

\author{
Osama Moaaz ${ }^{1}$, Choonkil Park ${ }^{2^{*}}$, Elmetwally M. Elabbasy ${ }^{1}$ and Waed Muhsin ${ }^{1}$
}

\section{"Correspondence:}

baak@hanyang.ac.kr

${ }^{2}$ Research Institute for Natural

Sciences, Hanyang University, Seoul

04763, Korea

Full list of author information is

available at the end of the article

\section{黛 Springer}

\begin{abstract}
In this work, we create new oscillation conditions for solutions of second-order differential equations with continuous delay. The new criteria were created based on Riccati transformation technique and comparison principles. Furthermore, we obtain iterative criteria that can be applied even when the other criteria fail. The results obtained in this paper improve and extend the relevant previous results as illustrated by examples.
\end{abstract}

MSC: $34 \mathrm{C} 10 ; 34 \mathrm{~K} 11$

Keywords: Deviating argument; Second order; Neutral differential equation; Oscillation

\section{Introduction}

The importance of studying delay differential equations DDEs is not limited to the theoretical side only, but the applications of this type of equations extend to many branches of applied science. In fact, the neutral DDEs arise in the examination of vibrating masses attached to an elastic bar, in the solution of variational problems with time delays, and in problems concerning electric networks containing lossless transmission lines (as in high speed computers), see $[1,2]$.

The great development in the study of asymptotic behavior of DDEs is easily noted in many works in recent times. Some of these works that are concerned with improving the oscillation criteria of DDEs are [3-6]. In addition, many improved methods and interesting results can be found in studies [7-23], which study the oscillatory behavior of the NDDEs of different order.

In this work, we discuss the oscillation properties of the second-order NDDE with distributed deviating arguments

$$
\left(r(t)\left((x+p \cdot x \circ \tau)^{\prime}(t)\right)^{\alpha}\right)^{\prime}+\Lambda[q \cdot f \circ x \circ g ; a, b](t)=0,
$$

(c) The Author(s) 2021. This article is licensed under a Creative Commons Attribution 4.0 International License, which permits use, sharing, adaptation, distribution and reproduction in any medium or format, as long as you give appropriate credit to the original author(s) and the source, provide a link to the Creative Commons licence, and indicate if changes were made. The images or other third party material in this article are included in the article's Creative Commons licence, unless indicated otherwise in a credit line to the material. If material is not included in the article's Creative Commons licence and your intended use is not permitted by statutory regulation or exceeds the permitted use, you will need to obtain permission directly from the copyright holder. To view a copy of this licence, visit http://creativecommons.org/licenses/by/4.0/ 
where $t \geq t_{0}$ and

$$
\Lambda[F ; a, b](t) \stackrel{\operatorname{def}}{=} \int_{a}^{b} F(t, s) \mathrm{d} s .
$$

Throughout this work, we assume that

$$
\aleph(t) \stackrel{\text { def }}{=}(x+p \cdot x \circ \tau)(t)
$$

and the following hypotheses hold:

$\left(\mathrm{H}_{1}\right) \alpha$ is a ratio of odd natural numbers, $r \in C\left(\left[t_{0}, \infty\right),(0, \infty)\right)$, and

$$
\int_{t_{0}}^{\infty} r^{-1 / \alpha}(s) \mathrm{d} s=\infty
$$

$\left(\mathrm{H}_{2}\right) p \in C\left(\left[t_{0}, \infty\right)\right), q \in C\left(\left[t_{0}, \infty\right) \times[a, b]\right), 0 \leq p(t)<1$, and $q(t, s)>0$ is not zero on any half line $\left[t_{*}, \infty\right) \times[a, b]$ for all $t_{*} \geq t_{0}$.

$\left(\mathrm{H}_{3}\right) \tau \in C\left(\left[t_{0}, \infty\right), \mathbb{R}\right), g \in C\left(\left[t_{0}, \infty\right) \times[a, b], \mathbb{R}\right), \tau(t) \leq t, \lim _{t \rightarrow \infty} \tau(t)=\infty, g(t, s) \leq t$, $\lim _{t \rightarrow \infty} g(t, s)=\infty$ for $s \in[a, b]$, and $g$ is strictly increasing with respect to $t$ and $s$ for all $s \in(a, b)$.

$\left(\mathrm{H}_{4}\right) f \in C((-\infty, \infty))$ and $f(x) / x^{\alpha} \geq \kappa$ for $x \neq 0$, where $\kappa$ is a positive constant.

For a solution of (1.1), we mean a function $x \in C\left(\left[t_{x}, \infty\right)\right), t_{x} \geq t_{0}$, which has the property $\aleph(t)$ and $r(t)\left(\aleph^{\prime}(t)\right)^{\alpha}$ are continuously differentiable for $t \in\left[t_{x}, \infty\right)$ and satisfies (1.1) on $\left[t_{x}, \infty\right)$. We focus only on the solutions of (1.1) which satisfy $\sup \left\{|x(t)|: t_{x} \leq t\right\}>0$ for $t \geq t_{x}$. A solution $x$ of (1.1) is called nonoscillatory if it is either eventually positive or eventually negative; otherwise it is called oscillatory.

In the next part of the introduction, we provide some related work that contributed to the development of the study of oscillatory behavior of NDDEs.

In 1985, Grammatikopoulos et al. [7] studied the asymptotic behavior of NDDE

$$
\left(x(t)+p(t) x\left(t-\tau_{0}\right)\right)^{\prime \prime}+q(t) x\left(t-g_{0}\right)=0 .
$$

They proved that all solutions of (1.3) are oscillatory if $p(t) \in[0,1]$ and

$$
\int_{t_{0}}^{\infty} q(v)\left(1-p\left(v-g_{0}\right)\right) \mathrm{d} v=\infty .
$$

However, Erbe et al. [8] established the oscillation condition when $q(t) \geq q_{0}>0, p(t) \in$ $\left[p_{1}, p_{2}\right]$ and $p(t)$ is not eventually negative. Posteriorly, Grace and Lalli [9] studied the oscillation of the NDDE

$$
\left(r(t)\left(x(t)+p(t) x\left(t-\tau_{0}\right)^{\prime}\right)^{\prime}+q(t) f\left(x\left(t-g_{0}\right)\right)=0,\right.
$$

under the condition

$$
\int_{t_{0}}^{\infty}\left(\rho(s) q(s)\left(1-p\left(s-g_{0}\right)\right)-\frac{r\left(s-g_{0}\right)\left(\rho^{\prime}(s)\right)^{2}}{4 \kappa \rho(s)}\right) \mathrm{d} s=\infty,
$$

where $\rho \in C^{1}\left(\left[t_{0}, \infty\right),(0, \infty)\right)$. 
In the previous decade, under the hypothesis $\tau \circ g=g \circ \tau$, Han et al. [10] presented the oscillation criteria for the NDDE

$$
\left.\left(r(t)(x(t)+p(t) x(\tau(t)))^{\prime}\right)^{\prime}+q(t) x(g(t))\right)=0 .
$$

In 2012, by using the Riccati transformation technique, Liu et al. [11] and Wu et al. [12] obtained the oscillation conditions for the NDDE

$$
\left(r(t)\left|w^{\prime}(t)\right|^{\alpha-1} w^{\prime}(t)\right)^{\prime}+q(t)|x(g(t))|^{\beta-1} x(g(t))=0,
$$

where $w(t):=x(t)+p(t) x(\tau(t)), \alpha \geq \beta, r^{\prime}(t)>0$, and $g^{\prime}(t)>0$. Based on establishing new comparison theorems that compare the second-order equation with a first-order DDE, Baculikova and Dzurina [13] studied the NDDE

$$
\left(r(t)\left((x(t)+p(t) x(\tau(t)))^{\prime}\right)^{\alpha}\right)^{\prime}+q(t) x^{\beta}(g(t))=0
$$

under the conditions

$$
0 \leq p(t) \leq p_{0}<\infty, \quad \tau^{\prime}(t) \geq \tau_{0}, \quad \text { and } \quad \tau \circ g=g \circ \tau
$$

Of interesting works recently, Moaaz et al. in $[14,15]$ studied the oscillatory properties of (1.4) and improved the results in [13].

For NDDE with distributed deviating arguments (1.1), Candan [16] studied the sufficient conditions for the oscillation of solutions.

In this work, we are creating an improved relationship between the corresponding function $\aleph$ and its first derivative. This new relationship helps us to get sharp criteria for testing the oscillation. Based on the Riccati transformation and comparison principles, we obtain new and different criteria for the oscillation of solutions of (1.1). The results obtained in this paper improve and extend the relevant previous results as illustrated by examples.

To prove our main results, we need the following auxiliary lemmas. The proof of the first lemma is similar to that of [13, Lemma 3] and hence we omit it.

Lemma 1.1 If $x$ is a positive solution of $(1.1)$ on $\left[t_{0}, \infty\right)$, then there exists $t_{1} \geq t_{0}$ such that

$$
\aleph(t)>0, \quad \aleph^{\prime}(t)>0, \quad\left(r(t)\left(\aleph^{\prime}(t)\right)^{\alpha}\right)^{\prime} \leq 0
$$

for $t \geq t_{1}$.

Lemma 1.2 ([14, Lemma 1.2]) Suppose that $F(s)=A s-B s^{(\alpha+1) / \alpha}$, where $A, B>0$ are constants. Then $F$ attains its maximum value on $\mathbb{R}$ at $s^{*}=(\alpha A /((\alpha+1) B))^{\alpha}$ and

$$
\max _{x \in r} F=F\left(s^{*}\right)=\frac{\alpha^{\alpha}}{(\alpha+1)^{\alpha+1}} \frac{A^{\alpha+1}}{B^{\alpha}} .
$$

Remark 1.1 All functional inequalities are assumed to hold eventually, that is, they are satisfied for all $t>t_{1}$, where $t_{1}$ is large enough. 


\section{Main results}

For convenience, we denote the class of all eventually positive solutions by $S^{+}$. Moreover, we assume the following notations: $\beta \stackrel{\text { def }}{=}(\alpha+1)^{\alpha+1}, g_{a}(t) \stackrel{\text { def }}{=} g(t, a)$,

$$
\begin{aligned}
& \Theta(t) \stackrel{\text { def }}{=} \Lambda\left[q \cdot(1-p \circ g)^{\alpha} ; a, b\right](t), \\
& \mu(t) \stackrel{\text { def }}{=} \int_{t_{1}}^{t} r^{-1 / \alpha}(\theta) \mathrm{d} \theta, \\
& \tilde{\mu}(t) \stackrel{\text { def }}{=} \mu(t)+\frac{\kappa}{\alpha} \int_{t_{1}}^{t} \mu(\theta) \mu^{\alpha}(g(\theta, a)) \Theta(\theta) \mathrm{d} \theta, \\
& \widehat{\mu}(t) \stackrel{\text { def }}{=} \exp \left(-\alpha \int_{g_{a}(t)}^{t} \frac{\mathrm{d} \theta}{\widetilde{\mu}(\theta) r^{1 / \alpha}(\theta)}\right),
\end{aligned}
$$

and $F_{+}(t) \stackrel{\text { def }}{=} \max \{0, F(t)\}$, where $t_{1} \geq t_{0}$.

The following theorem gives a criterion for the oscillation of (1.1), depending on the comparison with a first-order DDE.

Theorem 2.1 Every solution of (1.1) is oscillatory if the first-order DDE

$$
\phi^{\prime}(t)+\kappa \Theta(t) \tilde{\mu}^{\alpha}\left(g_{a}(t)\right) \phi\left(g_{a}(t)\right)=0
$$

is oscillatory.

Proof Assume the contrary that there is a nonoscillatory solution $x$ of (1.1). Then we can assume $x \in S^{+}$, and so $x(t), x(\tau(t))$, and $x(g(t, s))$ are positive for $t \geq t_{1} \geq t_{0}$ and $s \in[a, b]$. It follows from Lemma 1.1 that (1.5) holds. Using the fact that $r(t)\left(\aleph^{\prime}(t)\right)^{\alpha}$ is a nonincreasing function, we get

$$
\int_{t_{1}}^{t}\left(\frac{r(\theta)\left(\aleph^{\prime}(\theta)\right)^{\alpha}}{r(\theta)}\right)^{1 / \alpha} \mathrm{d} \theta \geq \mu(t)\left(r(t)\left(\aleph^{\prime}(t)\right)^{\alpha}\right)^{1 / \alpha}
$$

and hence

$$
\aleph(t) \geq \mu(t)\left(r(t)\left(\aleph^{\prime}(t)\right)^{\alpha}\right)^{1 / \alpha} .
$$

It follows from (1.1) and $\left(\mathrm{H}_{1}\right)$ that

$$
\left(r(t)\left(\aleph^{\prime}(t)\right)^{\alpha}\right)^{\prime} \leq-\kappa \int_{a}^{b} q(t, s) x^{\alpha}(g(t, s)) \mathrm{d} s .
$$

From the definition of $\aleph$, we have

$$
\begin{aligned}
x(g(t, s)) & =\aleph(g(t, s))-p(g(t, s)) x(\tau(g(t, s))) \\
& \geq \aleph(g(t, s))-p(g(t, s)) \aleph(\tau(g(t, s))) \\
& \geq \aleph(g(t, s))(1-p(g(t, s))),
\end{aligned}
$$


which with (2.3) gives

$$
\begin{aligned}
\left(r(t)\left(\aleph^{\prime}(t)\right)^{\alpha}\right)^{\prime} & \leq-\kappa \int_{a}^{b} q(t, s) \aleph^{\alpha}(g(t, s))[1-p(g(t, s))]^{\alpha} \mathrm{d} s \\
& \leq-\kappa \aleph^{\alpha}\left(g_{a}(t)\right) \int_{a}^{b} q(t, s)[1-p(g(t, s))]^{\alpha} \mathrm{d} s \\
& \leq-\kappa \Theta(t) \aleph^{\alpha}\left(g_{a}(t)\right),
\end{aligned}
$$

which is a direct result of the facts that $\aleph^{\prime}(t)>0$ and $\partial_{s} g(t, s)>0$. Combining

$$
\mu \frac{\mathrm{d}}{\mathrm{d} t}\left(r^{1 / \alpha} \aleph^{\prime}\right)^{\alpha}=\mu \alpha\left(r^{1 / \alpha} \aleph^{\prime}\right)^{\alpha-1}\left(r^{1 / \alpha} \aleph^{\prime}\right)^{\prime}
$$

and

$$
\frac{\mathrm{d}}{\mathrm{d} t}\left(\aleph-\mu r^{1 / \alpha} \aleph^{\prime}\right)=\aleph^{\prime}-\mu^{\prime} r^{1 / \alpha} \aleph^{\prime}-\mu\left(r^{1 / \alpha} \aleph^{\prime}\right)^{\prime}=-\mu\left(r^{1 / \alpha} \aleph^{\prime}\right)^{\prime},
$$

we get

$$
\frac{\mathrm{d}}{\mathrm{d} t}\left(\aleph-\mu r^{1 / \alpha} \aleph^{\prime}\right)=-\frac{1}{\alpha} \mu\left(r^{1 / \alpha} \aleph^{\prime}\right)^{1-\alpha} \frac{\mathrm{d}}{\mathrm{d} t}\left(r^{1 / \alpha} \aleph^{\prime}\right)^{\alpha}
$$

Thus, from (2.4), we find

$$
\frac{\mathrm{d}}{\mathrm{d} t}\left(\aleph(t)-\mu(t) r^{1 / \alpha}(t) \aleph^{\prime}(t)\right) \geq \frac{\kappa}{\alpha} \mu(t)\left(r^{1 / \alpha}(t) \aleph^{\prime}(t)\right)^{1-\alpha} \Theta(t) \aleph^{\alpha}\left(g_{a}(t)\right) .
$$

Integrating (2.5) from $t_{1} \rightarrow t$, we have

$$
\aleph(t) \geq \mu(t) r^{1 / \alpha}(t) \aleph^{\prime}(t)+\frac{\kappa}{\alpha} \int_{t_{1}}^{t} \mu(\theta) \Theta(\theta)\left(r^{1 / \alpha}(\theta) \aleph^{\prime}(\theta)\right)^{1-\alpha} \aleph^{\alpha}(g(\theta, a)) \mathrm{d} \theta .
$$

Now, we set $\phi(t):=r(t)\left(\aleph^{\prime}(t)\right)^{\alpha}$. Then, from (2.2) and (2.6), we obtain

$$
\begin{aligned}
\aleph(t) & \geq \mu(t) \phi^{1 / \alpha}(t)+\frac{\kappa}{\alpha} \int_{t_{1}}^{t} \mu(\theta) \Theta(\theta) \phi^{(1-\alpha) / \alpha}(\theta) \mu^{\alpha}(g(\theta, a)) \phi(g(\theta, a)) \mathrm{d} \theta \\
& \geq \mu(t) \phi^{1 / \alpha}(t)+\frac{\kappa}{\alpha} \int_{t_{1}}^{t} \mu(\theta) \Theta(\theta) \phi^{(1-\alpha) / \alpha}(\theta) \mu^{\alpha}(g(\theta, a)) \phi(\theta) \mathrm{d} \theta \\
& \geq \phi^{1 / \alpha}(t)\left(\mu(t)+\frac{\kappa}{\alpha} \int_{t_{1}}^{t} \mu(\theta) \mu^{\alpha}(g(\theta, a)) \Theta(\theta) \mathrm{d} \theta\right) \\
& \geq \widetilde{\mu}(t) \phi^{1 / \alpha}(t) .
\end{aligned}
$$

Combining (2.4) and (2.7), we have that $\phi$ is a positive solution of the first-order DD inequality

$$
\phi^{\prime}(t)+\kappa \Theta(t) \tilde{\mu}^{\alpha}\left(g_{a}(t)\right) \phi\left(g_{a}(t)\right) \leq 0 .
$$

From [24, Theorem 1], DDE (2.1) also has a positive solution, which is a contradiction. This contradiction completes the proof. 
Applying a well-known condition [25, Theorem 2.1.1] for oscillation of first-order DDE (2.1), we get immediately the following criteria for oscillation of (1.1).

Corollary 2.1 Every solution of (1.1) is oscillatory if one of the following conditions is satisfied:

$$
\liminf _{t \rightarrow \infty} \int_{g_{a}(t)}^{t} \tilde{\mu}^{\alpha}\left(g_{a}(\theta)\right) \Theta(\theta) \mathrm{d} \theta>\frac{1}{\kappa \mathrm{e}}
$$

or

$$
\limsup _{t \rightarrow \infty} \int_{g_{a}(t)}^{t} \tilde{\mu}^{\alpha}\left(g_{a}(\theta)\right) \Theta(\theta) \mathrm{d} \theta>\frac{1}{\kappa} \quad \text { and } \quad \partial_{t} g(t, s) \geq 0 .
$$

The next theorem gives another criterion for the oscillation of (1.1), depending on the Riccati transformation technique.

Theorem 2.2 Every solution of (1.1) is oscillatory if there is a positive function $\rho \in$ $C^{1}\left(\left[t_{0}, \infty\right)\right)$ satisfying

$$
\limsup _{t \rightarrow \infty} \int_{t_{1}}^{t}\left(\kappa \rho(\theta) \widehat{\mu}(\theta) \Theta(\theta)-\frac{r(\theta)\left(\rho_{+}^{\prime}(\theta)\right)^{\alpha+1}}{\beta \rho^{\alpha}(\theta)}\right) \mathrm{d} \theta=\infty,
$$

where $t_{1}$ is sufficiently large.

Proof Assume the contrary that there is a nonoscillatory solution $x$ of (1.1). Then we can assume $x \in S^{+}$, and so $x(t), x(\tau(t))$, and $x(g(t, s))$ are positive for $t \geq t_{1} \geq t_{0}$ and $s \in[a, b]$. It follows from Lemma 1.1 that (1.5) holds. Now, we set

$$
w \stackrel{\text { def }}{=} \rho r\left(\frac{\aleph^{\prime}}{\aleph}\right)^{\alpha} \text {. }
$$

Thus, we note that $w(t)>0$ for $t \geq t_{1}$. By differentiating $w$, we get

$$
\begin{aligned}
w^{\prime} & =\rho^{\prime} r\left(\frac{\aleph^{\prime}}{\aleph}\right)^{\alpha}+\rho \frac{\left(r \aleph^{\prime}\right)^{\prime}}{\aleph^{\alpha}}-\alpha \rho r\left(\frac{\aleph^{\prime}}{\aleph}\right)^{\alpha+1} \\
& =\frac{\rho^{\prime}}{\rho} w+\rho \frac{\left(r \aleph^{\prime}\right)^{\prime}}{\aleph^{\alpha}}-\alpha \rho^{-1 / \alpha} r^{-1 / \alpha} w^{1+1 / \alpha} .
\end{aligned}
$$

Next, as in the proof of Theorem 2.1, we obtain (2.4) and (2.7). Then, from (2.7), we obtain $\aleph(t) \geq \tilde{\mu}(t) r^{1 / \alpha}(t) \aleph^{\prime}(t)$ for $t \geq t_{1}$. Then, applying the Grönwall inequality, we find

$$
\aleph\left(g_{a}(t)\right) \geq \aleph(t) \exp \left[-\int_{g_{a}(t)}^{t} \tilde{\mu}^{-1}(\theta) r^{-1 / \alpha}(\theta) \mathrm{d} \theta\right]=\widehat{\mu}^{1 / \alpha}(t) \aleph(t)
$$

which with (2.4) gives

$$
\left(r(t)\left(\aleph^{\prime}(t)\right)^{\alpha}\right)^{\prime} \leq-\kappa \Theta(t)\left(\frac{\aleph\left(g_{a}(t)\right)}{\aleph(t)}\right)^{\alpha} \aleph^{\alpha}(t) \leq-\kappa \Theta(t) \widehat{\mu}(t) \aleph^{\alpha}(t) .
$$


Combining (2.12) and (2.13), we arrive at

$$
w^{\prime} \leq \frac{\rho_{+}^{\prime}}{\rho} w-\kappa \rho \Theta \widehat{\mu}(t)-\alpha \rho^{-1 / \alpha} r^{-1 / \alpha} w^{1+1 / \alpha} .
$$

Using Lemma 1.2 with $A=\rho_{+}^{\prime} / \rho$ and $B=\alpha(\rho r)^{-1 / \alpha}$, we get

$$
w^{\prime} \leq-\kappa \rho \Theta \widehat{\mu}+\frac{1}{\beta} r\left(\rho_{+}^{\prime}\right)^{\alpha+1} \rho^{-\alpha} .
$$

Integrating (2.15) from $t_{1} \rightarrow t$, we find

$$
w\left(t_{1}\right) \geq \int_{t_{1}}^{t}\left(\kappa \rho(\theta) \Theta(\theta) \widehat{\mu}(\theta)-\frac{1}{\beta} r(\theta)\left(\rho_{+}^{\prime}(\theta)^{\alpha+1}\right) \rho^{-\alpha}(\theta)\right) \mathrm{d} \theta
$$

which contradicts (2.10). This contradiction completes the proof.

It is easy to see that Corollary 2.1 cannot be applied in the case where

$$
\int_{g_{a}(t)}^{t} \tilde{\mu}^{\alpha}\left(g_{a}(\theta)\right) \Theta(\theta) \mathrm{d} \theta \leq \frac{1}{\kappa \mathrm{e}}
$$

However, if $x \in S^{+}$and (2.16) holds, then we can get a sharp estimate of $z(g(t)) / z(t)$. Thus, we can obtain a sharp criteria for the oscillation of (1.1).

Lemma 2.1 Assume that $x \in S^{+}$and

$$
\liminf _{t \rightarrow \infty} \int_{g_{a}(t)}^{t} \tilde{\mu}^{\alpha}\left(g_{a}(\theta)\right) \Theta(\theta) \mathrm{d} \theta \geq \delta
$$

for some $\delta>0$. Then

$$
\frac{r\left(g_{a}(t)\right)}{r(t)}\left(\frac{\aleph^{\prime}\left(g_{a}(t)\right)}{\aleph^{\prime}(t)}\right)^{\alpha} \geq \vartheta_{n}(\delta)
$$

for every $n \geq 0$, where

$$
\vartheta_{m}(\delta) \stackrel{\text { def }}{=} \begin{cases}1 & \text { if } m=0 \\ \exp \left(\delta \vartheta_{m-1}(\delta)\right) & \text { if } m>0\end{cases}
$$

Proof The proof of the first lemma is similar to that of [26, Lemma 1], and hence we omit it.

Theorem 2.3 Assume that (2.17) holds for some $\delta<0$. Every solution of (1.1) is oscillatory if there is a positive function $\rho \in C^{1}\left(\left[t_{0}, \infty\right)\right)$ satisfying

$$
\limsup _{t \rightarrow \infty} \int_{t_{1}}^{t}\left(\kappa \rho(\theta) \Theta(\theta)-\frac{\left(\rho_{+}^{\prime}(\theta)\right)^{\alpha+1} r\left(g_{a}(\theta)\right)}{\beta \vartheta_{m}(\delta) \rho^{\alpha}(\theta)\left(g_{a}^{\prime}(\theta)\right)^{\alpha}}\right)=\infty
$$

for some $m \geq 0$, where $\vartheta_{m}(\delta)$ is defined as (2.19). 
Proof Assume the contrary that there is a nonoscillatory solution $x$ of (1.1). Then we can assume $x \in S^{+}$, and so $x(t), x(\tau(t))$, and $x(g(t, s))$ are positive for $t \geq t_{1} \geq t_{0}$ and $s \in[a, b]$. It follows from Lemma 1.1 that (1.5) holds. As in the proof of Theorem 2.1, we obtain (2.4). Now, we set

$$
\omega \stackrel{\text { def }}{=} \rho r\left(\frac{\aleph^{\prime}}{\aleph\left(g_{a}\right)}\right)^{\alpha} \text {. }
$$

Thus, we note that $\omega(t)>0$ for $t \geq t_{1}$. By differentiating $\omega$ and using (2.4), we obtain

$$
\begin{aligned}
\omega^{\prime} & =\rho^{\prime} r\left(\frac{\aleph^{\prime}}{\aleph\left(g_{a}\right)}\right)^{\alpha}+\rho \frac{\left(r\left(\aleph^{\prime}\right)^{\alpha}\right)^{\prime}}{\aleph^{\alpha}\left(g_{a}\right)}-\alpha \rho r \frac{\left(\aleph^{\prime}\right)^{\alpha}}{\aleph^{\alpha+1}\left(g_{a}\right)} \aleph^{\prime}\left(g_{a}\right) g_{a}^{\prime} \\
& \leq \frac{\rho^{\prime}}{\rho} \omega-\kappa \rho \Theta-\alpha \rho r \frac{\left(\aleph^{\prime}\right)^{\alpha}}{\aleph^{\alpha+1}\left(g_{a}\right)} \aleph^{\prime}\left(g_{a}\right) g_{a}^{\prime} .
\end{aligned}
$$

Thus, it follows from Lemma 2.1 that

$$
\begin{aligned}
\omega^{\prime} & \leq \frac{\rho^{\prime}}{\rho} \omega-\kappa \rho \Theta-\alpha \rho \frac{r^{1+1 / \alpha} \vartheta_{n}^{1 / \alpha}(\delta)}{r^{1 / \alpha}\left(g_{a}\right)}\left(\frac{\aleph^{\prime}}{\aleph^{\alpha+1}\left(g_{a}\right)}\right)^{\alpha+1} g_{a}^{\prime} \\
& \leq \frac{\rho_{+}^{\prime}}{\rho} \omega-\kappa \rho \Theta-\alpha \frac{\vartheta_{n}^{1 / \alpha}(\delta) g_{a}^{\prime}}{\rho^{1 / \alpha} r^{1 / \alpha}\left(g_{a}\right)} \omega^{1+1 / \alpha} .
\end{aligned}
$$

Using Lemma 1.2 with $A=\rho_{+}^{\prime} / \rho$ and $B=\alpha \vartheta_{m}^{1 / \alpha}(\delta) /(\rho r(g))^{1 / \alpha}$, we get

$$
\omega^{\prime} \leq-\kappa \rho \Theta+\frac{\left(\rho_{+}^{\prime}\right)^{\alpha+1} r\left(g_{a}\right)}{\beta \vartheta_{m}(\delta) \rho^{\alpha}\left(g_{a}^{\prime}\right)^{\alpha}}
$$

Integrating (2.22) from $t_{1} \rightarrow t$, we find

$$
\omega\left(t_{1}\right) \geq \int_{t_{1}}^{t}\left(\kappa \rho(\theta) \Theta(\theta)-\frac{\left(\rho_{+}^{\prime}(\theta)\right)^{\alpha+1} r\left(g_{a}(\theta)\right)}{\beta \vartheta_{m}(\delta) \rho^{\alpha}(\theta)\left(g_{a}^{\prime}(\theta)\right)^{\alpha}}\right) \mathrm{d} \theta,
$$

which contradicts (2.20). This contradiction completes the proof.

\section{Further results}

It is easy to notice that (2.7) is a sharper estimate than (2.2) for the relationship between $\aleph$ and $\aleph^{\prime}$. By repeating the same steps that improved (2.2), we obtain iterative criteria that can be applied even when the other criteria fail.

Lemma 3.1 Assume that $x \in S^{+}$. Then

$$
\aleph(t) \geq U_{k}(t) r^{1 / \alpha}(t) \aleph^{\prime}(t)
$$

for $k=0,1, \ldots$, where $U_{0}(t):=\tilde{\mu}(t)$ and

$$
U_{k+1}(t):=\int_{t_{1}}^{t}\left(\frac{1}{r(s)} \exp \left(\int_{s}^{t} \kappa \Theta(\theta) U_{n}^{\alpha}\left(g_{a}(\theta)\right) \mathrm{d} \theta\right)\right)^{1 / \alpha} \mathrm{d} s
$$


Proof Assume that $x \in S^{+}$. Then $x(t), x(\tau(t))$, and $x(g(t, s))$ are positive for $t \geq t_{1} \geq t_{0}$ and $s \in[a, b]$. It follows from Lemma 1.1 that (1.5) holds. By induction, we will prove (3.1).

Now, as in the proof of Theorem 2.1, we obtain (2.4) and (2.7). From (2.7), we obtain

$$
\aleph \geq \widetilde{\mu}(t) r^{1 / \alpha} \aleph^{\prime}=U_{0} r^{1 / \alpha} \aleph^{\prime}
$$

Next, for $k=n$, we suppose that $\aleph \geq U_{n} r^{1 / \alpha} \aleph^{\prime}$. Hence, we get

$$
\aleph\left(g_{a}\right) \geq U_{n}\left(g_{a}\right) r^{1 / \alpha}\left(g_{a}\right) \aleph^{\prime}\left(g_{a}\right) \geq U_{n}\left(g_{a}\right) r^{1 / \alpha} \aleph^{\prime},
$$

which with (2.4) gives

$$
\kappa \Theta(t) \aleph^{\alpha}\left(g_{a}(t)\right)\left(r(t)\left(\aleph^{\prime}(t)\right)^{\alpha}\right)^{\prime}+\kappa \Theta(t) U_{n}^{\alpha}\left(g_{a}(t)\right) r(t)\left(\aleph^{\prime}(t)\right)^{\alpha} \leq 0 .
$$

Letting $H:=r\left(\aleph^{\prime}\right)^{\alpha},(3.3)$ reduces to

$$
H^{\prime}(t)+\kappa \Theta(t) U_{n}^{\alpha}\left(g_{a}(t)\right) H(t) \leq 0
$$

Applying the Grönwall inequality in (3.4), we find

$$
H(s) \geq H(t) \exp \left(\int_{s}^{t} \kappa \Theta(\theta) U_{n}^{\alpha}\left(g_{a}(\theta)\right) \mathrm{d} \theta\right)
$$

for $t \geq s \geq t_{1}$, and so

$$
\aleph^{\prime}(s) \geq r^{1 / \alpha}(t) \aleph^{\prime}(t)\left(\frac{1}{r(s)} \exp \left(\int_{s}^{t} \kappa \Theta(\theta) U_{n}^{\alpha}\left(g_{a}(\theta)\right) \mathrm{d} \theta\right)\right)^{1 / \alpha}
$$

Integrating (3.5) from $t_{1} \rightarrow t$, we see that

$$
\begin{aligned}
\aleph(t) & \geq r^{1 / \alpha}(t) \aleph^{\prime}(t) \int_{t_{1}}^{t}\left(\frac{1}{r(s)} \exp \left(\int_{s}^{t} \kappa \Theta(\theta) U_{n}^{\alpha}\left(g_{a}(\theta)\right) \mathrm{d} \theta\right)\right)^{1 / \alpha} \mathrm{d} s \\
& =U_{n+1}(t) r^{1 / \alpha}(t) \aleph^{\prime}(t) .
\end{aligned}
$$

This completes the proof.

Theorem 3.1 Assume that $U_{k}$ are defined as in Lemma 3.1. Every solution of (1.1) is oscillatory if

$$
\int_{t_{0}}^{\infty} \Theta(\theta) U_{k}(\theta) \mathrm{d} \theta=\infty
$$

for some $k=0,1, \ldots$

Proof Assume the contrary that there is a nonoscillatory solution $x$ of (1.1). Then we can assume $x \in S^{+}$, and so $x(t), x(\tau(t))$, and $x(g(t, s))$ are positive for $t \geq t_{1} \geq t_{0}$ and $s \in[a, b]$. It follows from Lemma 1.1 and 3.1 that (1.5) and (3.1) hold. As in the proof of Theorem 2.1, we obtain (2.4). 
Now, we define $w$ as in (2.11) with $\rho \equiv 1$. Proceeding as in the proof of Theorem 2.2 and substituting (2.7) with (3.1), we arrive at

$$
w^{\prime}(t)+\kappa \Theta(t) U_{k}(t)+\alpha r^{-1 / \alpha}(t) w^{1+1 / \alpha}(t) \leq 0
$$

or

$$
w^{\prime}(t)+\kappa \Theta(t) U_{k}(t) \leq 0 .
$$

Integrating (3.8) from $t_{1} \rightarrow t$, we get

$$
w(t) \leq w\left(t_{1}\right)-\kappa \int_{t_{1}}^{t} \Theta(\theta) U_{k}(\theta) \mathrm{d} \theta .
$$

Therefore, $w(t) \rightarrow-\infty$ as $t \rightarrow \infty$, which is a contradiction. This contradiction completes the proof.

Theorem 3.2 Assume that $U_{k}$ are defined as in Lemma 3.1. Every solution of (1.1) is oscillatory if

$$
\lim _{t \rightarrow \infty} \frac{1}{G(t)} \int_{t}^{\infty} r^{-1 / \alpha}(\theta) G^{1+1 / \alpha}(\theta) \mathrm{d} \theta>\frac{1}{\beta^{1 / \alpha}},
$$

where

$$
G(t) \stackrel{\text { def }}{=} \int_{t}^{\infty} \Theta(\theta) U_{k}(\theta) \mathrm{d} \theta .
$$

Proof Assume the contrary that there is a nonoscillatory solution $x$ of (1.1). Then we can assume $x \in S^{+}$, and so $x(t), x(\tau(t))$, and $x(g(t, s))$ are positive for $t \geq t_{1} \geq t_{0}$ and $s \in[a, b]$. By the same procedure as in the proof of Theorem 3.1, we arrive at (3.7). Then, integrating (3.7) from $t \rightarrow v$, we find

$$
\int_{t}^{v} \Theta(\theta) U_{k}(\theta) \mathrm{d} \theta+\alpha \int_{t}^{v} r^{-1 / \alpha}(\theta) w^{1+1 / \alpha}(\theta) \mathrm{d} \theta \leq w(t)-w(v) .
$$

Letting $v \rightarrow \infty$, we get

$$
G(t)+\alpha \int_{t}^{\infty} r^{-1 / \alpha}(\theta) w^{1+1 / \alpha}(\theta) \mathrm{d} \theta \leq w(t)
$$

or equivalently,

$$
1+\alpha \int_{t}^{\infty} r^{-1 / \alpha}(\theta) G^{1+1 / \alpha}(\theta)\left(\frac{w(\theta)}{G(\theta)}\right)^{1+1 / \alpha} \mathrm{d} \theta \leq \frac{w(t)}{G(t)} .
$$

If we set $\varrho=\inf _{t \geq t_{1}}(w(t) / G(t))$, then we note that $\varrho \geq 1$. However, from (3.9) and (3.11), we get

$$
\varrho \geq 1+\alpha\left(\frac{\varrho}{\alpha+1}\right)^{\frac{\alpha+1}{\alpha}}
$$


Therefore, relationship (3.12) can be modeled on the form

$$
\frac{\varrho}{\alpha+1} \geq \frac{1}{\alpha+1}+\frac{\alpha}{\alpha+1}\left(\frac{\varrho}{\alpha+1}\right)^{\frac{\alpha+1}{\alpha}}
$$

which contradicts the possible values of $\varrho$ and $\alpha$. This contradiction completes the proof.

For the following theorem, we need to define the sequence $\left\{\phi_{n}(t)\right\}_{n=0}^{\infty}$ as

$$
\phi_{n}(t) \stackrel{\text { def }}{=} \phi_{0}(t)+\alpha \int_{t}^{\infty} r^{-1 / \alpha}(\theta) \phi_{n-1}^{1+1 / \alpha}(\theta) \mathrm{d} \theta, \quad n=1,2,3, \ldots
$$

and

$$
\phi_{0}(t) \stackrel{\text { def }}{=} G(t)
$$

for all $t \geq t_{1} \geq t_{0}$, where $G$ is defined as in Theorem 3.2.

Lemma 3.2 Assume that $x \in S^{+}$and $w$ is defined as in (2.11) with $\rho \equiv 1$. Then $w(t) \geq \phi_{n}(t)$. In addition, there is a function $\phi \in C\left(\left[t_{1}, \infty\right),(0, \infty)\right)$ such that $\lim _{n \rightarrow \infty} \phi_{n}(t)=\phi(t)$ and

$$
\phi(t)=\phi_{0}(t)+\alpha \int_{t}^{\infty} r^{-1 / \alpha}(\theta) \phi^{1+1 / \alpha}(\theta) \mathrm{d} \theta .
$$

Proof Assume that $x \in S^{+}$. Then $x(t), x(\tau(t))$, and $x(g(t, s))$ are positive for $t \geq t_{1} \geq t_{0}$ and $s \in[a, b]$. By the same procedure as in the proof of Theorem 3.2, we arrive at (3.10), and hence

$$
\phi_{0}(t)=G(t) \leq w(t)
$$

Thus, from the definition of $\phi_{n}(t)$, we note that $w(t) \geq \phi_{n}(t)$ for all $n>1$ and $t \geq t_{1}$. Since $\left\{\phi_{n}(t)\right\}_{n=0}^{\infty}$ is an increasing sequence and bounded from above, $\phi_{n}(t)$ converges to $\phi(t)$. By using Lebesgue's monotone convergence theorem, if we take the limit of (3.13) as $n \rightarrow \infty$, then we obtain that (3.14) hold. The proof is complete.

Theorem 3.3 Assume that $U_{k}$ are defined as in Lemma 3.1. Every solution of (1.1) is oscillatory if

$$
\limsup _{t \rightarrow \infty} \phi_{n}(t)\left(\int_{t_{0}}^{t} r^{-\frac{1}{\alpha}}(s) \mathrm{d} s\right)^{\alpha}>1
$$

for some positive integers $n$.

Proof Assume the contrary that there is a nonoscillatory solution $x$ of (1.1). Then we can assume $x \in S^{+}$, and so $x(t), x(\tau(t))$, and $x(g(t, s))$ are positive for $t \geq t_{1} \geq t_{0}$ and $s \in[a, b]$. Now, we define $w$ as in (2.11) with $\rho \equiv 1$. From the fact that $\left(r(s)\left(\aleph^{\prime}(s)\right)^{\alpha}\right)^{\prime} \leq 0$, we have

$$
\aleph(t)=\aleph\left(t_{1}\right)+\int_{t_{1}}^{t} \frac{1}{r^{1 / \alpha}(\theta)}\left(r(\theta)\left(\aleph^{\prime}(\theta)\right)^{\alpha}\right)^{1 / \alpha} \mathrm{d} \theta
$$




$$
\geq\left(r(t)\left(\aleph^{\prime}(t)\right)^{\alpha}\right)^{1 / \alpha} \int_{t_{1}}^{t} \frac{1}{r^{1 / \alpha}(\theta)} \mathrm{d} \theta
$$

which with (2.11) gives

$$
\begin{aligned}
w(t) & =r(t)\left(\aleph^{\prime}(t)\right)^{\alpha} \aleph^{-\alpha}(t) \\
& \leq r(t)\left(\aleph^{\prime}(t)\right)^{\alpha}\left(\left(r(t)\left(\aleph^{\prime}(t)\right)^{\alpha}\right)^{1 / \alpha} \int_{t_{1}}^{t} \frac{1}{r^{1 / \alpha}(\theta)} \mathrm{d} \theta\right)^{-\alpha} \\
& =\left(\int_{t_{1}}^{t} \frac{1}{r^{1 / \alpha}(\theta)} \mathrm{d} \theta\right)^{-\alpha} .
\end{aligned}
$$

Thus,

$$
w(t)\left(\int_{t_{0}}^{t} \frac{1}{r^{1 / \alpha}(\theta)} \mathrm{d} \theta\right)^{\alpha} \leq\left(\frac{\int_{t_{0}}^{t} r^{-1 / \alpha}(\theta) \mathrm{d} \theta}{\int_{t_{1}}^{t} r^{-1 / \alpha}(\theta) \mathrm{d} \theta}\right)^{\alpha} \leq 1
$$

for $t \geq t_{1}$. Taking into account Lemma 3.2, we get a contradiction with (3.15). This contradiction completes the proof.

\section{Examples}

In this section, we apply our main results to some special cases of (1.1) and also compare our results with the previous related results.

Example 4.1 Consider the second-order NDDE

$$
\left(\left(\left(x(t)+p_{0} x \circ \tau\right)^{\prime}\right)^{\alpha}\right)^{\prime}+\Lambda\left[\frac{q_{0}}{t^{\alpha+1}} \cdot(x \circ g)^{\alpha} ; a, 1\right]=0,
$$

where $p_{0} \in[0,1), q_{0}>0, \delta \in[0,1), \tau(t)=\eta t, \eta \in(0,1)$, and $g(t, s)=s t$ for $s \in[a, 1]$. Obviously, we see that

$$
\begin{aligned}
& b=1, \quad r(t) \equiv 1, \quad p(t) \equiv p_{0}, \quad q(t, s) \equiv q_{0} / t^{\alpha+1} \quad \text { and } \\
& f(x) \equiv x^{\alpha}, \quad \text { with constant } \kappa=1 .
\end{aligned}
$$

Therefore, it is easy to verify that

$$
\begin{aligned}
& \Theta(t)=\frac{q_{0}}{t^{\alpha+1}}(1-a)\left(1-p_{0}\right)^{\alpha}, \quad \mu(t)=t, \quad \tilde{\mu}(t)=(1+\lambda) t, \\
& \widehat{\mu}(t)=a^{1 /(1+\lambda)}, \quad \text { and } \quad G(t)=\frac{\lambda a^{\alpha /(1+\lambda)}}{a^{\alpha}} \frac{1}{t^{\alpha}},
\end{aligned}
$$

where

$$
\lambda \stackrel{\text { def }}{=} \frac{1}{\alpha} a^{\alpha}(1-a)\left(1-p_{0}\right)^{\alpha} q_{0}
$$


Next, to apply Corollary 2.1, we must first check either condition (2.9) or (2.8). By substitution and a simple computation, we obtain

$$
\begin{aligned}
\liminf _{t \rightarrow \infty} \int_{g_{a}(t)}^{t} \tilde{\mu}^{\alpha}\left(g_{a}(\theta)\right) \Theta(\theta) \mathrm{d} \theta & =\alpha \lambda(1+\lambda)^{\alpha} \liminf _{t \rightarrow \infty} \int_{a t}^{t} \frac{1}{\theta} \mathrm{d} \theta \\
& =\alpha \lambda(1+\lambda)^{\alpha} \ln \frac{1}{a}
\end{aligned}
$$

Thus, by using Corollary 2.1, (4.1) is oscillatory if

$$
\alpha \lambda(1+\lambda)^{\alpha} \ln \frac{1}{a}>\frac{1}{\mathrm{e}} .
$$

On the other hand, condition (3.9) with $k=1$ reduces to

$$
\begin{aligned}
\lim _{t \rightarrow \infty} \frac{1}{G(t)} \int_{t}^{\infty} r^{-1 / \alpha}(\theta) G^{1+1 / \alpha}(\theta) \mathrm{d} \theta & =\left(\frac{\lambda}{\alpha} \frac{a^{\alpha+\alpha /(1+\lambda)}}{\alpha}\right)^{1 / \alpha} \lim _{t \rightarrow \infty} t^{\alpha} \int_{t}^{\infty} \frac{1}{\theta^{\alpha+1}} \mathrm{~d} \theta \\
& =\frac{1}{\alpha}\left(\frac{\lambda}{\alpha} \frac{a^{\alpha+\alpha /(1+\lambda)}}{\alpha}\right)^{1 / \alpha}>\frac{1}{\beta^{1 / \alpha}}
\end{aligned}
$$

From Theorem 3.2, equation (4.1) is oscillatory if

$$
\lambda a^{\alpha+\alpha /(1+\lambda)}>\frac{\alpha^{\alpha+2}}{\beta} .
$$

Example 4.2 Consider the second-order NDDE

$$
\left(\left((x(t)+(1-\delta) x(\eta t))^{\prime}\right)^{\alpha}\right)^{\prime}+\frac{q_{0}}{t^{\alpha+1}} x^{\alpha}(\lambda t)=0,
$$

where $\delta \in(0,1], q_{0}>0$, and $\eta, \lambda \in(0,1)$. Obviously, we see that

$$
\begin{aligned}
& a=0, \quad b=1, \quad r(t) \equiv 1, \quad p(t) \equiv 1-\gamma, \quad q(t, s) \equiv q_{0} / t^{\alpha+1}, \quad \tau(t) \equiv \eta t, \\
& g(t, s) \equiv \lambda t \quad \text { and } \quad f(x) \equiv x^{\alpha}, \quad \text { with constant } \kappa=1 .
\end{aligned}
$$

Therefore, it is easy to verify that

$$
\Theta(t)=\delta^{\alpha} q_{0} \frac{1}{t^{\alpha+1}}, \quad \mu(t)=t, \quad U_{0}(t)=\left(1+\frac{1}{\alpha} \lambda^{\alpha} \delta^{\alpha} q_{0}\right) t
$$

and

$$
U_{1}(t)=\lambda^{\alpha /\left(1+\frac{1}{\alpha} \lambda^{\alpha} \delta^{\alpha} q_{0}\right)} .
$$

From Theorem 3.2, equation (4.1) is oscillatory if

$$
q_{0} \delta^{\alpha} \lambda^{\alpha /\left(1+\frac{1}{\alpha} \lambda^{\alpha} \delta^{\alpha} q_{0}\right)}>\frac{\alpha^{\alpha+1}}{\beta} .
$$


Remark 4.1 Consider the special case of (4.2) where $\delta=1, \alpha=1 / 3$, and $\lambda=0.9$. The oscillation criteria in [13, Corollary 2] and [17, Corollary 2.1] reduce to $q_{0}>3.61643$ and $q_{0}>1.92916$, respectively. However, (4.3) reduces to $q_{0}>0.16131$. So, our results improve and extend some of the previous results.

\section{Conclusion}

The oscillation theory of DDEs has many applications in applied sciences. Thus, studying the oscillation of the solutions of these equations has practical importance besides the theoretical importance. In this study, we obtained different oscillation criteria with different techniques. These new criteria enable us to test the oscillation of a class of NDDEs with continuous delay. Our results extended to recently published works [14, 15], and also improved [13, 17].

Modeling by fractional-order differential equations has more advantages than by classical integer-order ones as it considers the effects of existence of time memory or long-range space interactions. So, it would be interesting to extend the results of this paper to the fractional delay differential equations. Moreover, it is interesting to study the periodicity behavior of solutions of the studied equation as an extension of the works [19, 23].

\section{Acknowledgements}

The author is grateful to the editors and two anonymous referees for a very thorough reading of the manuscript and for pointing out some inaccuracies.

\section{Funding}

This work was supported by the Basic Science Research Program through the National Research Foundation of Korea funded by the Ministry of Education, Science and Technology (NRF-2017R1D1A1B04032937).

\section{Availability of data and materials}

Not applicable.

\section{Competing interests}

The authors declare that they have no competing interests.

\section{Authors' contributions}

The authors equally conceived of the study, participated in its design and coordination, drafted the manuscript, participated in the sequence alignment, and read and approved the final manuscript.

\section{Author details}

${ }^{1}$ Department of Mathematics, Faculty of Science, Mansoura University, 35516 Mansoura, Egypt. ${ }^{2}$ Research Institute for Natural Sciences, Hanyang University, Seoul 04763, Korea.

\section{Publisher's Note}

Springer Nature remains neutral with regard to jurisdictional claims in published maps and institutional affiliations.

Received: 1 April 2020 Accepted: 19 March 2021 Published online: 26 March 2021

\section{References}

1. Bainov, D.D., Mishev, D.P.: Oscillation Theory for Neutral Differential Equations with Delay. Hilger, New York (1991)

2. Hale, J.K.: Theory of Functional Differential Equations. Springer, New York (1977)

3. Agarwal, R.P., Shieh, S.L., Yeh, C.C.: Oscillation criteria for second order retarded differential equations. Math. Comput. Model. 26, 1-11 (1997)

4. Kusano, T., Naito, Y:: Oscillation and nonoscillation criteria for second order quasilinear differential equations. Acta Math. Hung. 76, 81-99 (1997)

5. Chatzarakis, G.E., Moaaz, O., Li, T., Qaraad, B.: Some oscillation theorems for nonlinear second-order differential equations with an advanced argument. Adv. Differ. Equ. 2020, 160 (2020)

6. Moaaz, O., Muhib, A.: New oscillation criteria for nonlinear delay differential equations of fourth order. Appl. Math. Comput. 377, $125192(2020)$

7. Grammatikopoulos, M.K., Ladas, G., Meimaridou, A.: Oscillation of second order neutral delay differential equation. Rad. Math. 1, 267-274 (1985)

8. Erbe, L.H., Kong, Q., Zhang, B.G.: Oscillation Theory for Functional Differential Equations. Dekker, New York (1995)

9. Grace, S.R., Lalli, B.S.: Oscillation of nonlinear second order neutral delay differential equations. Rad. Math. 3,77-84 (1987) 
10. Han, Z., Li, T., Sun, S., Chen, W.: On the oscillation of second-order neutral delay differential equations. Adv. Differ. Equ. 2010, Article ID 289340 (2010)

11. Liu, H., Meng, F., Liu, P.: Oscillation and asymptotic analysis on a new generalized Emden-Fowler equation. Appl. Math. Comput. 219, 2739-2748 (2012)

12. $W u, Y ., Y u, Y ., Z$ Zhang, J., Xiao, J.: Oscillation criteria for second order Emden-Fowler functional differential equations of neutral type. Appl. Math. Comput. 219, 2739-2748 (2012)

13. Baculikova, B., Dzurina, J.: Oscillation theorems for second-order nonlinear neutral differential equations. Comput. Math. Appl. 62, 4472-4478 (2011)

14. Moaaz, O.: New criteria for oscillation of nonlinear neutral differential equations. Adv. Differ. Equ. 2019, 484 (2019)

15. Moaaz, O., Anis, M., Baleanu, D., Muhib, A.: More effective criteria for oscillation of second-order differential equations with neutral arguments. Mathematics 8, 986 (2020)

16. Candan, T.: Oscillatory behavior of second order nonlinear neutral differential equations with distributed deviating arguments. Appl. Math. Comput. 262, 199-203 (2015)

17. Grace, S.R., Dzurina, J., Jadlovska, I., Li, T.: An improved approach for studying oscillation of second-order neutral delay differential equations. J. Inequal. Appl. 2018, 193 (2018)

18. Karpuz, B., Santra, S.S.: Oscillation theorems for second-order nonlinear delay differential equations of neutral type. Hacet. J. Math. Stat. 48(3), 633-643 (2019)

19. Lu, S., Yu, X.: Periodic solutions for second order differential equations with indefinite singularities. Adv. Nonlinear Anal. 9(1), 994-1007 (2020)

20. Moaaz, O., Elabbasy, E.M., Qaraad, B.: An improved approach for studying oscillation of generalized Emden-Fowler neutral differential equation. J. Inequal. Appl. 2020, 69 (2020)

21. Moaaz, O., Furuichi, S., Muhib, A.: New comparison theorems for the $n$th order neutral differential equations with delay inequalities. Mathematics 8(3), 454 (2020)

22. Moaaz, O., Cesarano, C., Muhib, A.: Some new oscillation results for fourth-order neutral differential equations. Eur. J. Pure Appl. Math. 13(2), 185-199 (2020)

23. Zhao, H.Y.: Pseudo almost periodic solutions for a class of differential equation with delays depending on state. Adv. Nonlinear Anal. 9(1), 1251-1258 (2020)

24. Philos, C.: On the existence of nonoscillatory solutions tending to zero at $\infty$ for differential equations with positive delay. Arch. Math. (Basel) 36, 168-178 (1981)

25. Ladde, G., Lakshmikantham, V., Zhang, B.: Oscillation Theory of Differential Equations with Deviating Arguments. Dekker, New York (1987)

26. Zhang, B.G., Zhou, Y.: The distribution of zeros of solutions of differential equations with a variable delay. J. Math. Anal. Appl. 256, 216-228 (2001)

\section{Submit your manuscript to a SpringerOpen ${ }^{\circ}$ journal and benefit from:}

- Convenient online submission

- Rigorous peer review

- Open access: articles freely available online

- High visibility within the field

- Retaining the copyright to your article

Submit your next manuscript at $\gg$ springeropen.com 